\title{
Construction and evaluation of an expression vector allowing the stable expression of foreign antigens in a Salmonella typhimurium vaccine strain
}

\author{
Edwin J. Tijhaar*, Yan Zheng-Xin ${ }^{\dagger}$, Jos A. Karlas*, Thomas F. Meyer ${ }^{\dagger}$, \\ Marij J. Stukart*f, Albert D.M.E. Osterhaus ${ }^{\S}$ and Frits R. Mooi ${ }^{\| \infty}$
}

Salmonella strains have great potential as live carriers of heterologous antigens to induce immunity against a variety of infectious diseases. However, the amount of heterologous antigen required to induce an adequate immune response may be toxic for the bacterium and result in cell death, overattenuation or loss of expression of the heterologous antigen. To solve this problem an expression vector was developed with a strong promoter located on a DNA fragment which is inverted at random. Antigen is only expressed in one particular orientation of the promoter. Thus a bacterial population harbouring the plasmid will consist of a subpopulation which does not produce heterologous antigen, and is therefore not affected in growth, persistence and dissemination within the host. Further, this non-producing population will continuously segregate antigen-producing bacteria. To evaluate the system, CtxB was used as a model antigen. Analysis of the plasmid DNA isolated from Salmonella revealed a selection against the promoter orientation that directs transcription of the ctxB gene. In spite of this, the vector was stably maintained in vivo and induced CtxB-specific IgA and $\lg G$ in mice. These results indicate that this kind of expression vector may offer a solution to the problem of unstable expression of foreign antigens in live bacterial vaccine strains.

Keywords: Salmonella typhimurium; plasmid stability; invertible promoter; CtxB

\begin{abstract}
Attenuated Salmonella strains expressing heterologous antigens have been shown to be able to induce mucosal, humoral and cell-mediated immunity against these antigens ${ }^{1}$. With respect to cell-mediated immunity, both class I and II MHC-restricted cytotoxic T cells directed against heterologous antigens have been detected ${ }^{2-4}$. Furthermore, in animal models protective immunity has been induced with a number of Salmonella-recombinant strains against bacterial, viral and protozoal infections ${ }^{4-6}$.
\end{abstract}

* Laboratory of Immunobiology, National Institute of Public Health and Environmental Protection, PO Box 1, 3720 BA Bilthoven, The Netherlands. 'Department of Infection Biology, Max Planck Institute for Biology, Spemannstrasse 34, 7400 Tübingen, Germany. ‡Present address: Department of Pathology, Free University Hospital, de Boelelaan 1117, 1081 HV Amsterdam, The Netherlands. "Department of Molecular Microbiology, National Institute of Public Health and Environmental Protection, PO Box 1, 3720 BA Bilthoven, The Netherlands. `Department of virology, Erasmus University Rotterdam, Dr. Molewaterplein 50, 3015 GE Rotterdam, The netherlands. ${ }^{\infty}$ To whom correspondence should be addressed. (Received 25 August 1993; revised 2 December 1993; accepted 9 December 1993)

0264-410X/94/11/1004-08

(c) 1994 Butterworth-Heinemann Ltd

1004 Vaccine 1994 Volume 12 Number 11
Live Salmonella vaccine strains have been accepted for use in humans. An oral human typhoid vaccine based on Salmonella typhi strain Ty21a, a galE mutant, has been licensed in many countries after it was shown to be efficacious in field trials? ${ }^{7}$. Other more defined attenuated Salmonella strains have been constructed, such as aro $A$ and $c y a, c r p$ mutants. These strains have been shown to be safe and immunogenic in a number of animal models. Salmonella strains attenuated by aro mutations have been shown to be safe even in immune-suppressed animals ${ }^{1}$. $S$, typhi aro $A$, pur $A$ and $S$. typhi aro $C, D$ strains have undergone phase 1 clinical trials in humans ${ }^{8,9}$.

Clearly, Salmonella strains have great potential as live carriers to induce immunity against a variety of infectious diseases. However, the amount of heterologous antigen required to obtain an adequate immune response is often toxic for the bacterium, and this forms a major obstacle to the use of recombinant Salmonella strains. In order to solve this problem, an expression system was developed, based on an invertible promoter, which results in high-level expression of the antigen of interest in only a minor part of the bacterial population ${ }^{10}$. If the amount of antigen produced is toxic for the bacterium, it will stop growing, but the non-producing part of the 
population will continue to grow and segregate new antigen producers. Here we describe the construction of this expression vector and its evaluation in a mouse model using the B subunit of cholera toxin $(\mathrm{CtxB})$ as a model antigen.

\section{MATERIALS AND METHODS}

\section{Bacterial strains, plasmids and media}

Escherichia coli strain DH5 $\alpha$ (BRL Life Technologies, Breda, The Netherlands) was used as a host for all plasmid constructions. Before introduction into the Salmonella typhimurium vaccination strain SL326111, plasmids were passaged through the restriction-deficient, modification-proficient $S$. typhimurium strain SL5283. All bacteria were grown in NZCYM ${ }^{12}$ aerobically at $37^{\circ} \mathrm{C}$, unless indicated otherwise. Ampicillin was used at $100 \mu \mathrm{g} \mathrm{ml}^{-1}$.

\section{DNA manipulations}

Isolation of plasmid DNA, preparation of DNA fragments and ligations were carried out using standard methods ${ }^{12}$. Plasmids were introduced into bacteria by electroporation using the Biorad Gene Pulser (Bio-Rad, Richmond, CA, USA) as follows: overnight cultures were diluted 1:100 in NZCYM and grown aerobically at $37^{\circ} \mathrm{C}$ to mid-log phase. The cells were subsequently harvested by centrifugation and washed twice with ice-cold distilled water. Finally, the cells were resuspended to $10^{10}$ cells $\mathrm{ml}^{-1}$ in distilled water containing $10 \%(\mathrm{w} / \mathrm{v})$ glycerol, frozen in dry ice and stored at $-70^{\circ} \mathrm{C}$ until use. For electroporation, DNA was precipitated, resuspended in distilled water and subsequently mixed with $10^{9}$ cells in an ice-cooled cuvette and electroporated at $2.5 \mathrm{kV}$, $25 \mu \mathrm{F}$ and $200 \Omega$. Immediately after electroporation $1 \mathrm{ml}$ of NZCYM was added and the cells were subsequently incubated aerobically for $30 \mathrm{~min}$ at $37^{\circ} \mathrm{C}$. Aliquots were plated on NZCYM-agar plates containing ampicillin and incubated at $37^{\circ} \mathrm{C}$.

\section{Immunization of mice and in vivo stability of pYZ17}

For oral immunization, bacteria from logarithmically growing cultures were harvested by centrifugation, washed in phosphate-buffered saline (PBS) and resuspended to a cell concentration of $2 \times 10^{10}$ cells $\mathrm{ml}^{-1}$ in $10 \%$ $\mathrm{NaHCO}_{3}$. Subsequently, 6-week-old female Balb/c mice received two successive oral immunizations with $10^{10}$ bacteria at days 1 and 4 followed by two successive booster immunizations with the same number of bacteria at days 32 and 36 . At days 11, 18, 25, 32 and 60 two mice per group were bled and their spleens and guts were removed. Gut-washes were obtained by washing the complete gut-segment distal from the stomach with $1 \mathrm{ml}$ PBS, centrifuging the samples for $10 \mathrm{~min}$ at 10000 rev $\min ^{-1}$ and collecting the supernatants. Gut-washes and sera were stored at $-20^{\circ} \mathrm{C}$ until tested by ELISA. The spleens were homogenized and the stability of pYZ17 was determined by comparing the number of viable bacteria that could be recovered on McConkey agar plates with and without ampicillin $\left(100 \mu \mathrm{g} \mathrm{ml}^{-1}\right)$. Essentially the same procedure was followed for intraperitoneal (i.p.) immunization, except that bacteria were suspended in PBS to a cell concentration of $2 \times 10^{6}$ cells $\mathrm{ml}^{-1}$, and mice were immunized with $10^{6}$ bacteria intraperitoneally (i.p.) at days 4 and 32 .

\section{ELISA}

B-lactamase ELISA. $\beta$-lactamase (TEM R $\mathrm{R}^{+}$, Boehringer Mannheim, Germany) was diluted in $0.1 \mathrm{M}$ phosphate buffer, $\mathrm{pH} 8.1$, to a protein concentration of $2.5 \mu \mathrm{g} \mathrm{ml}^{-1}$ and adsorbed onto 96-well high-binding microtitre plates (Costar E.I.A./R.I.A. plate HB, Cambridge, USA) by overnight incubation at $4^{\circ} \mathrm{C}$. After washing with demineralized water containing $0.05 \%$ Tween-80, plates were blocked with PBS containing 5\% fetal calf serum (FCS), 5\% NaCl and $0.1 \%$ Tween- 20 . Plates were washed with $0.05 \%$ Tween- 80 containing demineralized water and each well was incubated with $100 \mu \mathrm{l}$ dilutions of the sera or gut-washes. After incubation for $1 \mathrm{~h}$ at $37^{\circ} \mathrm{C}$, the plates were washed twice and the wells were incubated with goat anti-mouse IgG or IgA HRP-conjugates (Southern Biotechnology Inc., Birmingham, UK) diluted 8000 times. Binding of the conjugate was detected by addition of $100 \mu \mathrm{l}$ of a TMB/DONS-based substrate solution to each well. After $10 \mathrm{~min}$ at room temperature, $100 \mu \mathrm{l} 2 \mathrm{M} \mathrm{H}_{2} \mathrm{SO}_{4}$ was added to stop the colour reaction. The absorbance at $450 \mathrm{~nm}$ was determined in a Titertek Multiscan (Titertek, ICN Biomedicals, Amsterdam, The Netherlands). The titre was defined as the highest dilution of test samples at which the $A_{450}$ was higher than the mean $A_{450}+$ three times s.d. of the corresponding samples from $\mathrm{Balb} / \mathrm{c}$ mice immunized identically with SL3261 without pYZ17.

LPS-ELISA. The ELISA for LPS differed from the $\beta$-lactamase ELISA only in the coating step, which was performed in $0.11 \mathrm{M}$ acetate buffer, pH 5.5, containing $5.0 \mu \mathrm{g} \mathrm{ml}^{-1} S$. typhimurium LPS (Sigma, cat. no. L6511, Axel, The Netherlands) and the anti-IgG and $\operatorname{IgA}$ HRP-conjugate dilutions employed (1:4000). The titre was defined as the highest serum dilution at which the $A_{450}$ was higher than the mean $A_{450}+$ three times standard deviation (s.d.) of preimmune sera.

CtxB-ELISA. CtxB (List Biological Labs, Campbell, CA, USA) was diluted in PBS to a concentration of $5.0 \mu \mathrm{g} \mathrm{ml}^{-1}$ and adsorbed to the microtitre plates. The used anti-IgG and IgA conjugates were diluted 10000 and 4000 times respectively. All the other conditions were as described for the $\beta$-lactamase ELISA.

\section{Western blot analysis of recombinant bacteria}

Overnight cultures were diluted ten times in NZCYM and incubated aerobically until the absorbance of the culture at $550 \mathrm{~nm}$ reached 1.0. Aliquots of bacteria were pelleted, resuspended in $100 \mu 1$ sample buffer ${ }^{13}$ and boiled for $20 \mathrm{~min}$. Samples of $10 \mu \mathrm{l}$ were separated on $15 \%$ SDS-PAGE and subsequently transferred to nitrocellulose. After blocking with $0.5 \%$ Tween- 80 in PBS for $30 \mathrm{~min}$ the blots were incubated at room temperature with a goat-anti-CtxB serum (List Biological Labs, Campbell, CA, USA) diluted 500 times in PBS containing $0.5 \%$ Tween- 80 and $0.5 \%$ low-fat milk powder. After washing three times over a period of $30 \mathrm{~min}$ in PBS containing $0.5 \%$ Tween- 80 , the blot was incubated for $1 \mathrm{~h}$ at room temperature with horseradish peroxidaseconjugated protein A (Amersham, Den Bosch, The Netherlands) diluted 2500 times with $0.5 \%$ Tween- 80 in PBS. After three washings, the blot was developed in a TMB/DONS-based substrate. 


\section{RESULTS}

\section{Construction and characterization of pYZ17}

Plasmid pYZ17 is an expression plasmid with an invertible promoter based on the bacteriophage $\mathrm{Mu}$ Gin system. The Gin invertase of phage $\mathrm{Mu}$ initiates spontaneous $\mathrm{G}$ segment inversion on phage $\mathrm{Mu}$ genome, which takes place between two 34 bp inverted repeat (IR) sequences ${ }^{14}$. The size of the invertible segment is not a crucial factor in the inversion process, a $G$ segment as small as $40 \mathrm{bp}$ is still inverted, and the left ( $\mathrm{L}$ ) and right (R) IR sequences are equivalent in terms of their roles in the recombination process and can therefore substitute for each other ${ }^{15}$. Based on these observations, an invertible promoter can be constructed by inserting a promoter fragment between two identical IR sequences. Plasmid pYZ17 is a derivative of pYZ16a (Yan and Meyer, manuscript in preparation), which has the following features; a promoterless DNA fragment coding for phage $\mathrm{Mu}$ Gin invertase, a clts 857 gene fragment coding for temperature-sensitive lambda cl repressor, a fd transcriptional terminator downstream and an $\operatorname{rrnB}$ T1 transcriptional terminator upstream of clts857 fragment, a promoterless cholera toxin subunit B fragment sequence, origin of replication and ampicillin resistance gene from pBR328. Plasmid pYZ17 (Figure 1) was constructed by introducing an $X h o I$ IR-P $P_{L}-I R$ module into the single $X$ hoI site on pYZ16a. To construct the IR- $P_{\mathbf{L}}-\mathrm{IR}$ module, the lambda leftward promoter $\mathrm{P}_{\mathbf{L}}$ was isolated from pLC2833 16 as an $\sim 250 \mathrm{bp}$ XholI fragment and ligated at both ends with a synthetic IR sequence flanked by $B c l$ I/ Xhol sites: 5'-GATCATTTACCGTTTCCTGTAAACCGAGGTTTTGGATAAC-3'. After ligation, the IR- $P_{L}-I R$ module was inserted into pYZ16a. In the resulting plasmid, pYZ17, antigen (CtxB) expression can only occur in one orientation of the $P_{\mathbf{L}}$ promoter (designated the 'ON' orientation). Besides a correct orientation of the $\mathrm{P}_{\mathrm{L}}$ promoter, expression of $\mathrm{Ctx} \mathrm{B}$ requires the bacteria containing $\mathrm{pYZ17}$ to be grown at temperatures higher than $28^{\circ} \mathrm{C}$ to inactivate the thermosensitive repressor of $P_{L}$

Expression of CtxB was analysed on Western blots, after growing strain SL3261 harbouring pYZ17 at 28 and $37^{\circ} \mathrm{C}$. As expected, CtxB could not be detected when bacteria were grown at $28^{\circ} \mathrm{C}$, but was clearly induced at $37^{\circ} \mathrm{C}$ (Figure 2).

If there is no selection against a particular orientation of the $\mathrm{P}_{\mathrm{L}}$ promoter, one expects that in a bacterial population habouring $\mathrm{pYZ} 17,50 \%$ of the plasmids will contain the $P_{L}$ promoter in the 'oN' orientation. However, if transcription driven by the $P_{L}$ promoter in a particular orientation (most probably the $\mathrm{ON}$ orientation) is detrimental, the percentage of plasmids with this orientation will be smaller than $50 \%$. To estimate the fraction of $\mathrm{pYZ} 17$ molecules in the bacterial population which contained $P_{L}$ in the oN orientation, plasmid DNA was isolated from bacteria grown at 28 and $37^{\circ} \mathrm{C}$, and subsequently digested with EcoRI. Plasmid pYZ17 contains two EcoRI restriction sites (Figure 1), one of which is located between the two inverted repeats (IR) just downstream of the $P_{L}$ promoter. Digestion of $p Y Z 17$ with EcoRI should result in fragments of 5.0 and $1.9 \mathrm{~kb}$ for plasmids containing the $\mathbf{P}_{\mathbf{L}}$ promoter in the 'OFF' orientation and 4.7 and $2.2 \mathrm{~kb}$ for the oN orientation. Analysis of EcoRI-digested pYZ17 DNA derived from bacteria grown at $37^{\circ} \mathrm{C}$ revealed that a small fraction of

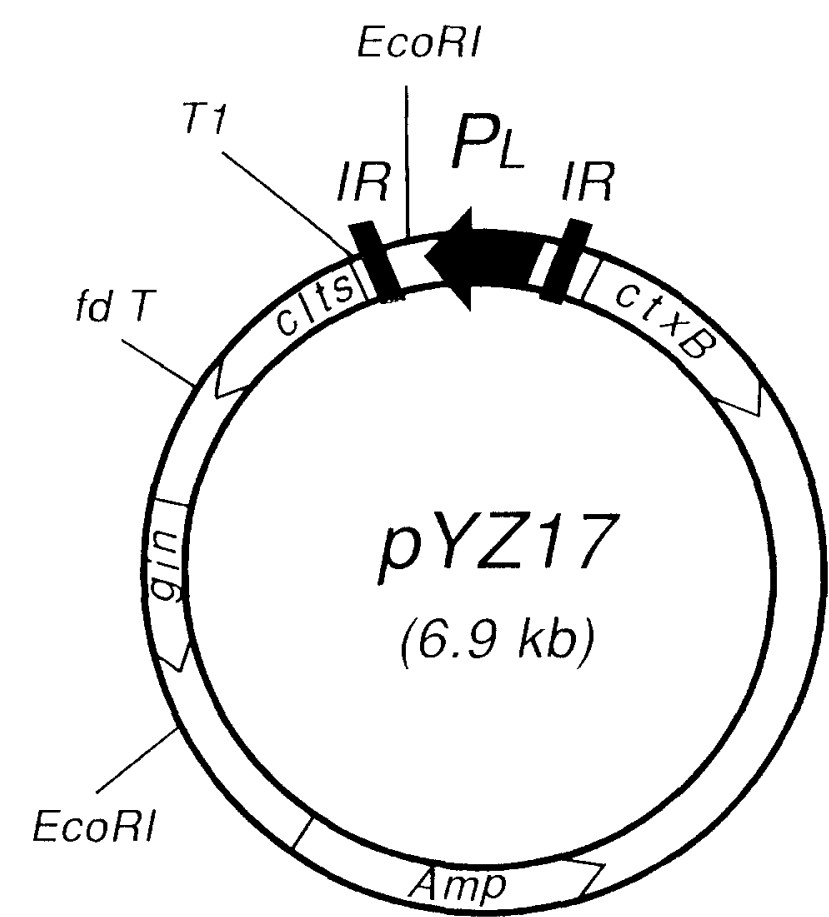

Figure 1 Plasmid pYZ17 containing an invertible promoter for CtxB expression. $P_{L}$, leftward promoter of bacteriophage lambda; IR, inverted repeat; $T 1$, rrnB $T 1$ transcription terminator; $f d T$, transcription terminator fd T; amp, ampicillin resistance gene; gin, invertase gene; clts, phage lambda temperature-sensitive repressor protein gene; ctxB cholera toxin $\mathrm{B}$ subunit gene. The two EcoRI sites which can be used to determine the orientation of the $P_{L}$ promoter are indicated. Shown is pYZ17 with the $P_{L}$ promoter in the OFF orientation

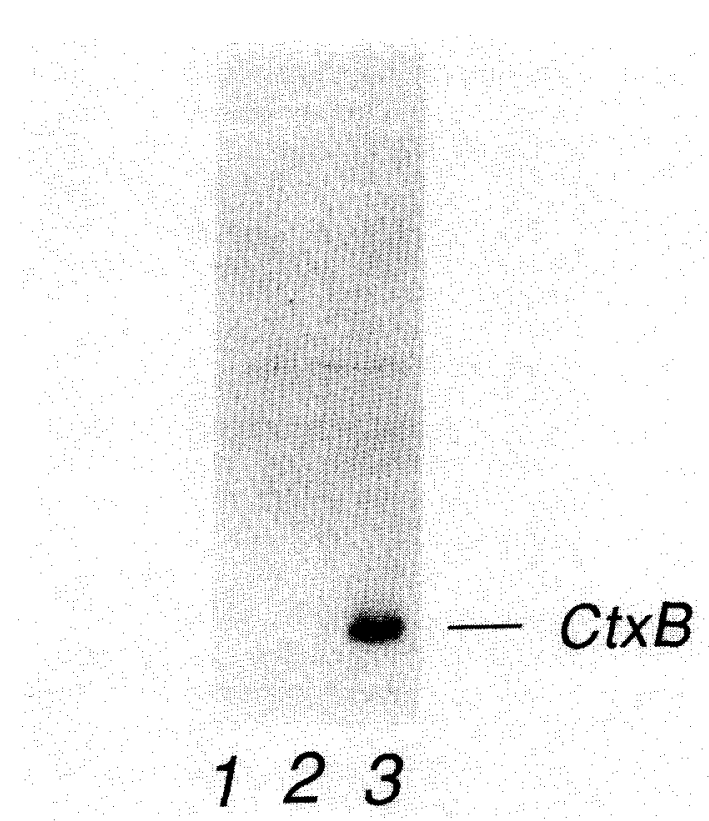

Figure $2 \mathrm{CtxB}$ expression by SL3261(pYZ17) at 28 and $37^{\circ} \mathrm{C}$. Total cell lysates containing equal quantities of bacteria were subjected to SDS-PAGE, followed by Western blotting using a CtxB-specific serum. Lane $1, S L 3261$ grown at $37^{\circ} \mathrm{C}$; lane 2, SL3261 (pYZ17) grown at $28^{\circ} \mathrm{C}$; lane $3, S L 3261$ ( $P Y Z 17$ ) grown at $37^{\circ} \mathrm{C}$

pYZ17 plasmids contained $\mathrm{P}_{L}$ in the oN orientation, suggesting that $\mathrm{P}_{\mathrm{L}}$-driven transcription towards the $\operatorname{ct} x B$ gene is selected against (Figure 3). Unexpectedly, when cells were grown at $28^{\circ} \mathrm{C}$ most pYZ17 molecules were also found to contain the $P_{L}$ promoter in the oFF orientation. This suggests that even the low transcription 
level from the $\mathrm{P}_{\mathrm{L}}$ promoter at $28^{\circ} \mathrm{C}$ is sufficient to select against the oN-form of $\mathrm{pYZ} 17$.

\section{Immunogenicity and stability of SL3261 (pYZ17)}

To determine whether pYZ17 was stably maintained in vivo, mice were infected i.p. with SL3261(pYZ17), and bacteria were recovered from the spleens after 1,2 and 3 weeks to determine the fraction of SL3261 cells still containing pYZ17 (Figure 4). During this period, pYZ17 appeared to be completely stable. Five bacterial colonies recovered from the spleens were tested for expression of $\mathrm{CtxB}$, and turned out to produce the same amount of $\mathrm{CtxB}$ as the original strain used for infection (results not shown).

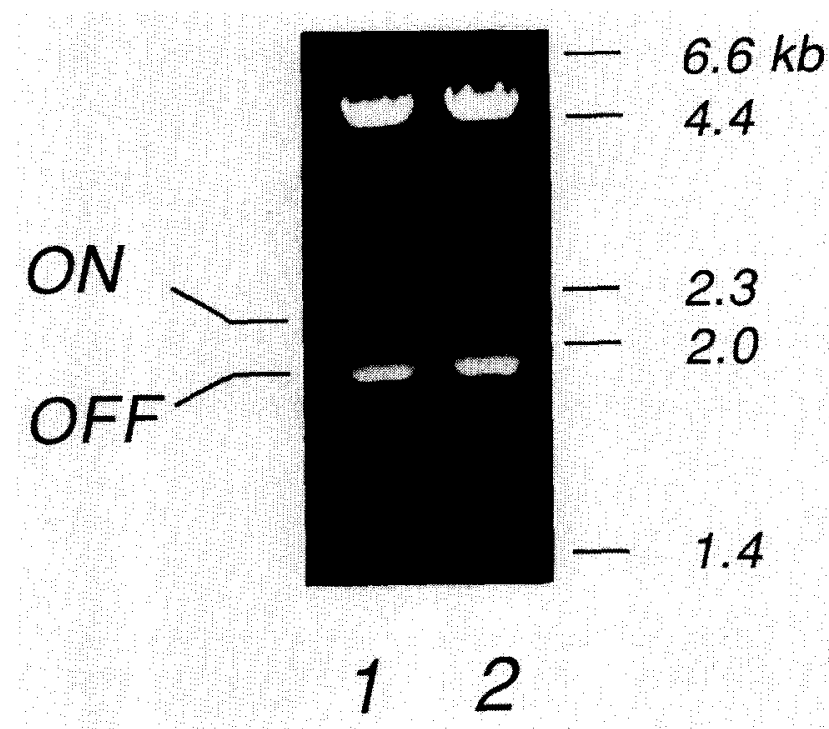

Figure 3 Restriction analysis of pYZ17, derived from strains grown at 28 or $37^{\circ} \mathrm{C}$. Plasmid DNA was isolated from $S L 5283$ (pYZ17) grown at 28 or $37^{\circ} \mathrm{C}$, digested with $E C O R I$ and subjected to electrophoresis on a $1 \%$ agarose gel. Fragments characteristic for plasmids containing the $P_{L}$ promoter in ON and OFF orientation are indicated

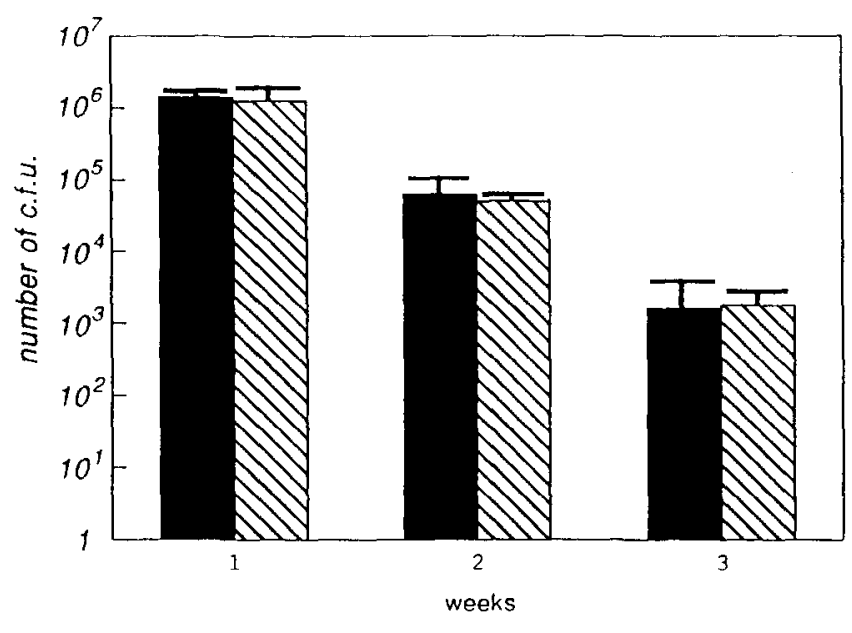

Figure 4 Stability of pYZ17 in vivo; $10^{6}$ cells of strain SL3261(pYZ17) were administered i.p. to Balb/c mice. After 1, 2 and 3 weeks, mice were killed and the number of bacteria present in the spleen still containing pYZ17 was compared with the total number of bacteria by plating on McConkey agar plates with (hatched bars) and without (filled bars) ampicillin, respectively. Represented are the geometric means (bars) and the corresponding maximum numbers of colony-forming units
The immunogenicity of SL3261(pYZ17) was determined in mice after oral and i.p. immunization. After oral immunization, CtxB-specific IgG and IgA could be detected in serum (Figure $5 a$ and $b$, respectively). Furthermore, IgA could also be detected in gut washes (Figure 6a), indicating that, besides a systemic immune response, a mucosal immune response was induced. The IgG titres remained high for up to 4 weeks after immunization. The IgA titres reached their maximum value within 1 week after immunization. During the following weeks these titres decreased, but they were restored by a booster at week 4 .

In contrast to the oral immunization, no $\mathrm{CtxB}$-specific antibodies could be detected after i.p. immunization (Figure 5). The difference in immune response against CtxB observed after oral and i.p. immunization could be due to the nature of $\mathrm{CtxB}$, which is known to be a very effective mucosal immunogen ${ }^{17,18}$. The immunization with $\mathrm{CtxB}$ by the oral route may be inherently more effective than immunization by the i.p. route. Alternatively the i.p. immunization could have been less effective because a lower number of cells was administered than in oral immunization. To discriminate between these possibilities, the antibody responses of the orally and i.p. immunized mice against two other bacterial antigens were compared. These antigens were lipopolysaccharide (LPS) and $\beta$-lactamase, which are expressed on the surface and in the periplasmic space of SL3261(pYZ17), respectively. Both oral and i.p. immunization resulted in the appearance of LPS-specific $\operatorname{IgG}$ and $\operatorname{IgA}$ in serum (Figure $7 a$ and $b$, respectively). Furthermore, both routes of immunization induced a LPS-specific secretory IgA response (Figure $6 b$ ). Both oral and i.p. immunization resulted in a $\beta$-lactamase-specific IgG response in serum (Figure 8), although the i.p. route was clearly more effective than the oral route. Specific IgA against $\beta$-lactamase could not be detected in the sera or in the gut washes after oral or i.p. immunization (results not shown).

These results indicate that for LPS and $\beta$-lactamase i.p. immunization was at least as effective as oral immunization. Thus the difference between the response against $\mathrm{CtxB}$ after oral and i.p. immunization is most probably due to the fact that $\mathrm{CtxB}$ is much more immunogenic when presented orally than when presented parentally.

\section{DISCUSSION}

Attenuated Salmonella strains have great potential as carriers for the delivery of heterologous antigens to the immune system. However, construction of Salmonellarecombinant strains expressing the heterologous antigen at levels high enough to induce protective immunity without affecting the ability of the strain to invade and persist in the host, is a major problem. Expression of (high levels of) heterologous antigens may be detrimental or toxic for the bacteria, resulting in loss of expression in vivo ${ }^{19,20}$ or overattenuation. In particular, loss of expression due to plasmid segregation has been a problem $^{21}$. For this reason much effort has been put into developing systems which increase the stability of the DNA expressing the heterologous antigen. Curtiss et al. ${ }^{22-24}$ have developed a host-vector system in which the antigen expressing DNA is located on a plasmid which contains a gene, coding for aspartate 

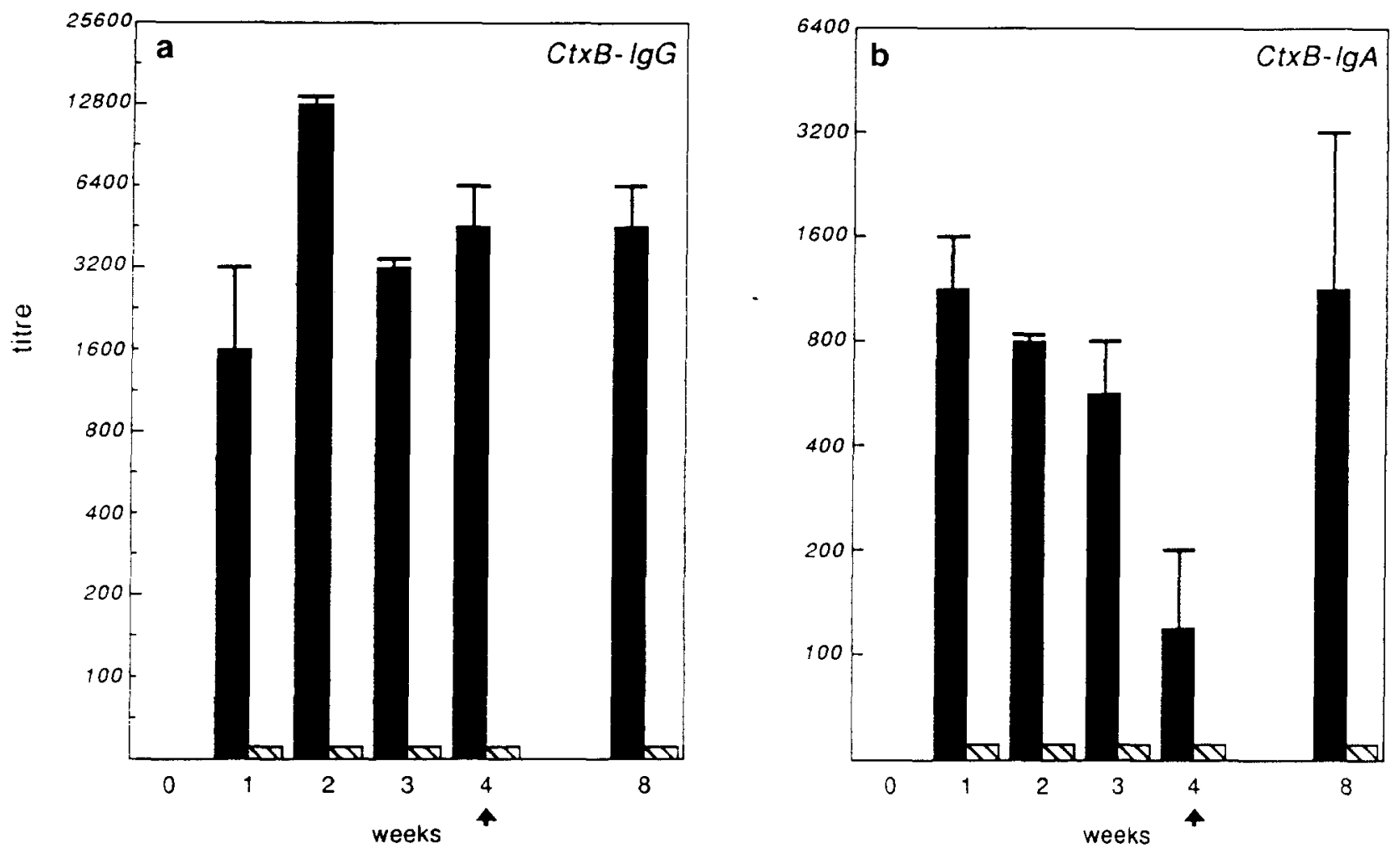

Figure 5 Antibody response to CtxB in sera after oral (filled bars) and i.p. (hatched bars) immunization of mice with SL3261(pYZ17). SL3261(pYZ17) was administered to Balb/c mice at weeks 0 and 4 . Every week, two mice of both groups were bled and their anti-CtxB serum titres were determined. Represented are geometric means (bars) and the corresponding maximum titres. The arrow indicates the booster immunization. (a) Anti-CtxB IgG response; (b) anti-CtxB IgA response
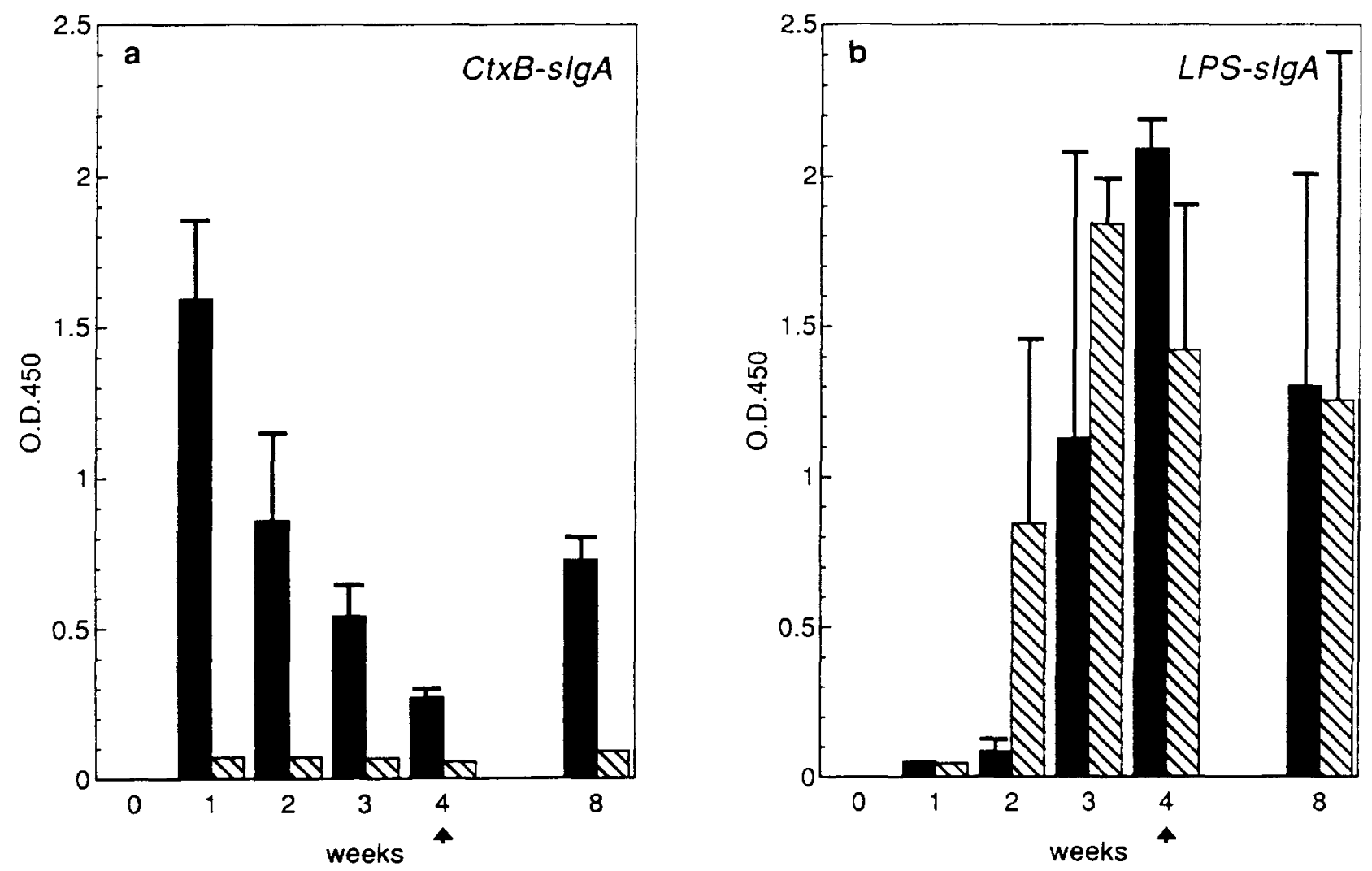

Figure 6 IgA response of four times diluted gut-washes after oral (filled bars) and i.p. (hatched bars) immunization of mice with SL3261(pYZ17). SL3261(pYZ17) was administered to Balb/c mice at weeks 0 and 4. Every week, two mice of both groups were killed and their gut washes were tested in ELISA. Represented are geometric means (bars) and the corresponding maximum $A_{450}$ values. The arrow indicates the booster immunization. (a) Anti-CtxB response; (b) anti-LPS response 

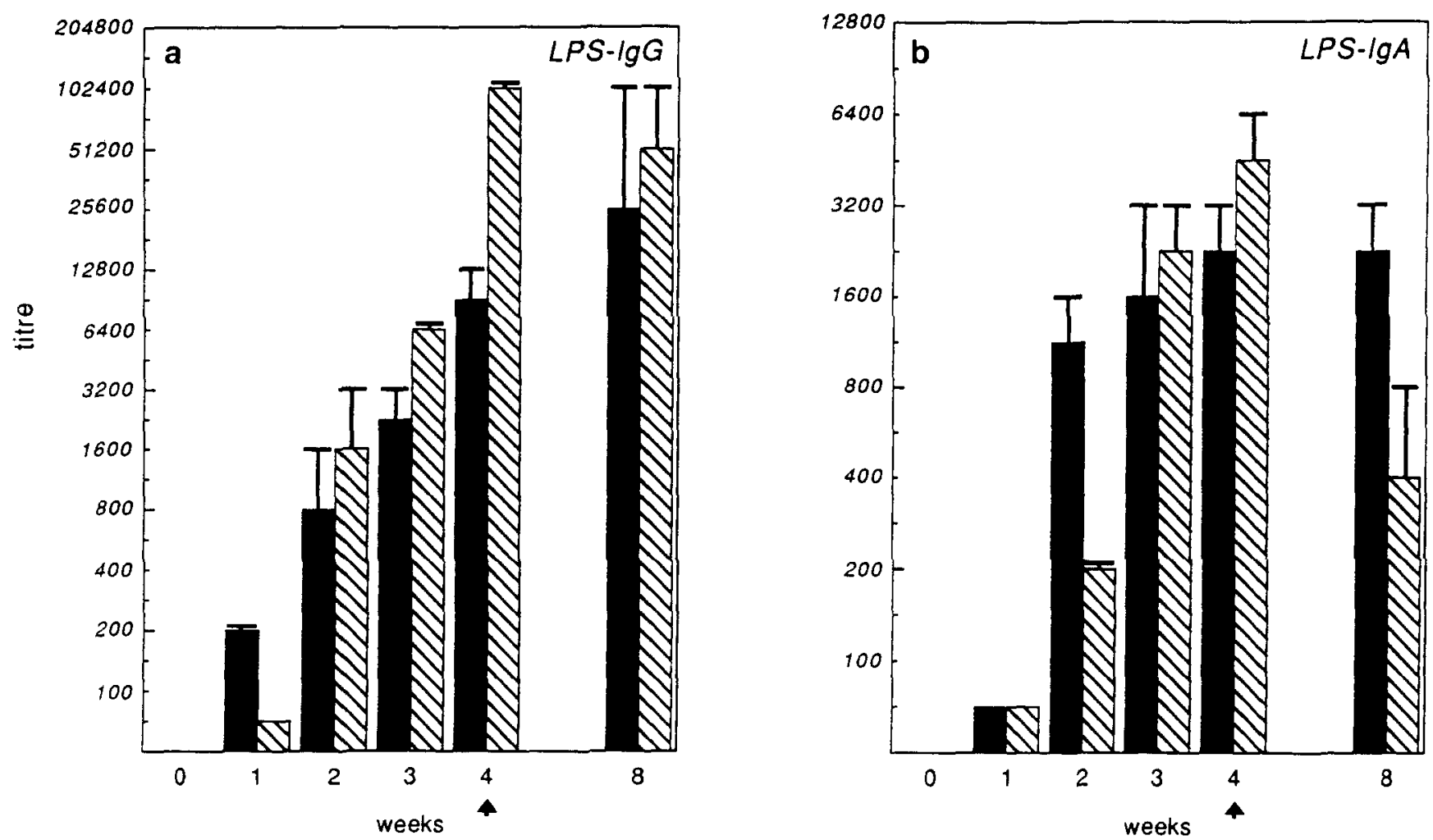

Figure 7 Antibody response to LPS in sera after oral (filled bars) and i.p. (hatched bars) immunization of mice with SL3261(pYZ17). SL3261(pYZ17) was administered to Balb/c mice at weeks 0 and 4. Every week, two mice of both groups were bled and their anti-LPS serum titres were determined. Represented are geometric means (bars) and the corresponding maximum titres. The arrow indicates the booster immunization. (a) Anti-LPS IgG response; (b) anti-LPS IgA response

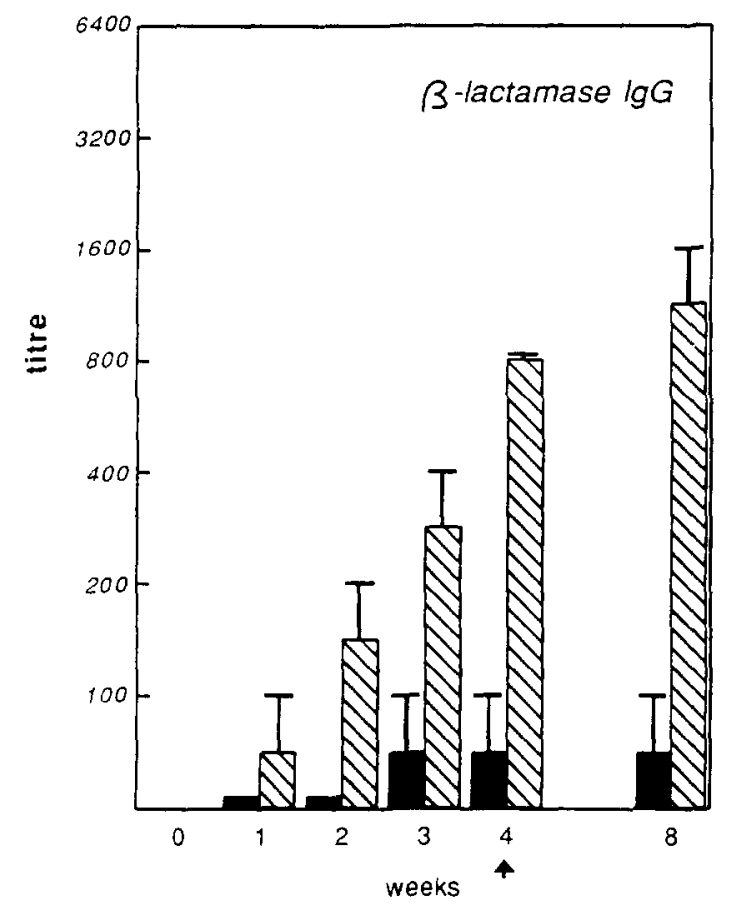

Figure 8 lgG response to $\beta$-lactamase in sera after oral (filled bars) and i.p. (hatched bars) immunization of mice with SL3261(pYZ17). SL3261 (pYZ17) was administered to Balb/c mice at weeks 0 and 4. Every week, two mice of both groups were bled and their anti- $\beta$-lactamase serum titres were determined. Values shown are geometric means with their maximum titres. The arrow indicates the booster immunization

$\beta$-semialdehyde dehydrogenase, which is essential for growth of the host strain. The stability of the antigen-expressing DNA may also be increased by integrating it into the chromosome $\mathrm{e}^{3,25,26}$. Although these systems have proven to be very useful for the stable expression of a number of antigens, they do not allow expression of antigens at levels which inhibit cell growth, because this will inevitably result in mutational alterations to reduce expression ${ }^{25}$. This is a major problem, because many heterologous antigens are toxic for $S$. typhimurium due to different codon usage or the presence of toxic (e.g. hydrophobic) sequences.

Deliberate reduction of the expression to a level high enough to induce an adequate immune response and low enough not to affect the fitness of the carrier strain is another strategy to obtain stable antigen expression. However, this balance between the immunogenicity and toxicity will depend on the nature of the antigen and will have to be determined anew every time another antigen is used. For some antigens this approach will be ineffective because, to induce an adequate immune response, they require expression at levels that are toxic to the producing vaccine strain.

We have developed a vector for stable expression of antigens, based on an invertible $P_{L}$ promoter. The essence of the vector is that it directs expression of an antigen in only a minor part of the bacterial population. If production of the antigen is lethal, the antigen-producing bacteria will stop dividing. However, the non-producing part of the population will continue to grow and segregate antigen-producing cells due to inversion of the $P_{\mathbf{L}}$ promoter. In this way, dissemination and invasion of the total bacterial population will not be affected by antigen production (Figure 9).

To evaluate our system, we used $\mathrm{CtxB}$ as a model antigen. A plasmid, designated pYZ17, was constructed in which expression of the $\operatorname{ct} x B$ gene is controlled by an invertible $P_{L}$ promoter. It appeared that $\mathrm{pYZ17}$ was stably maintained in vitro and in vivo. Analysis of $\mathrm{pYZ} 17$ isolated from SL5283 revealed that the copy number of 


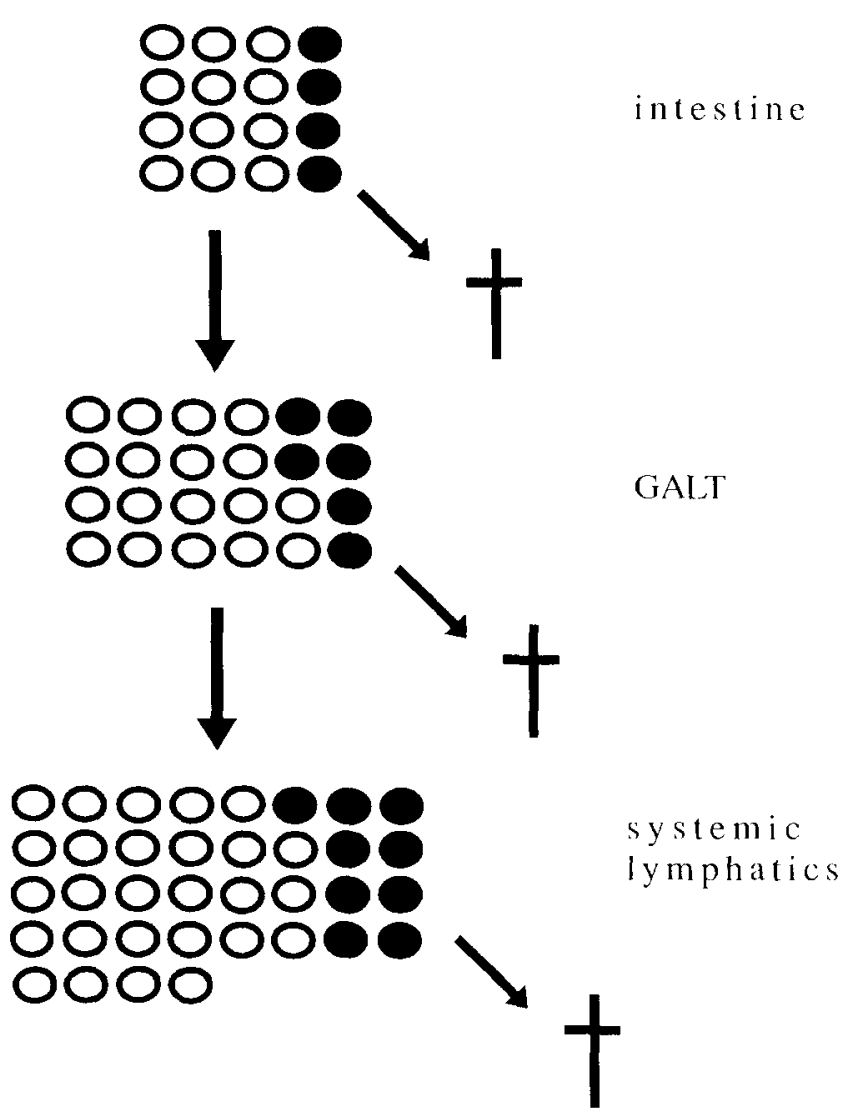

Figure 9 Model illustrating the course of an infection with SL3261 expressing foreign antigens due to promoter inversions. Circles indicate bacteria that do (O) and do not $(O)$ produce antigen. In this figure it is assumed that in every generation, $25 \%$ of the bacteria will produce toxic amounts of antigen due to inversion of the $P_{L}$ promoter and stop growing. After oral administration the bacteria will start to colonize the gut-associated lymphoid tissue (GALT). The bacteria that produce the foreign antigen will stop growing and may even lyse. The non-producing bacteria will continue the infection and may colonize the lymph nodes, spleen and liver without being affected by production of the antigen. During the infection antigen-producing cells will constantly be generated due to inversion of the $P_{L}$ promoter

the on plasmids was substantially lower than the copy number of the OFF plasmids. The apparent low copy number of pYZ17-ON may be caused by effects at the level of cell growth and/or plasmid replication. If the production of $\mathrm{CtxB}$ inhibits cell growth, bacteria containing higher numbers of pYZ17-oN will be outgrown by bacteria containing lower numbers of this plasmid. However, the observation that the oN/OFF ratio is not affected by the large difference in $\mathrm{CtxB}$ production at 28 and $37^{\circ} \mathrm{C}$, nor by deletion of the $\operatorname{ct} \times B$ gene (result not shown) indicates that this effect is of minor importance. The alternative explanation for the low copy number of $\mathrm{pYZ} 17-\mathrm{ON}$, i.e. an effect on plasmid replication, seems more likely. For example, transcription from the $P_{L}$ promoter in the oN orientation may interfere with plasmid replication. A similar phenomenon has been described by Strueber and Bujard ${ }^{27}$. The observation that the growth temperature does not affect the oN/OFF ratio suggests that even the leaky transcription at $28^{\circ} \mathrm{C}$ is sufficient to interfere with plasmid replication. We are currently addressing these questions.

In spite of the low copy number of pYZ17-on, strains isolated from the spleens of mice still produced CtxB. Apparently pYZ17-ON is stably maintained in the bacterial population due to continuous replenishment from pYZ17-ofF. Furthermore, strain SL3261(pYZ17) was able to induce an immune response against $\mathrm{CtxB}$. The induced CtxB-specific IgG and IgA titres in the serum and the presence of $\operatorname{IgA}$ in gut washes demonstrated that $\mathrm{Ctx} B$ was efficiently presented in the immune system after oral administration of the $S$. typhimurium strain SL3261 harbouring pYZ17 to Balb/c mice. The i.p. route turned out to be ineffective to induce an immune response against $\mathrm{CtxB}$. This phenomenon does not generally apply to other antigens as demonstrated by the fact that antibodies to LPS and the pYZ17-encoded $\beta$-lactamase were induced more efficiently by the i.p. than by the oral route. Others have also found that i.p. immunization in general induces stronger immune responses than oral administration of recombinant Salmonella strains ${ }^{28}$. The $\mathrm{CtxB}$-pentamer binds specifically to the GM1 gangliosides that are abundantly present on mucosal epithelial cells ${ }^{17,18}$. This results in a very efficient presentation of $\mathrm{CtxB}$ to the immune system by the oral route. We speculate that the amount of $\mathrm{Ctx} B$ expressed by SL3261(pYZ17) was too low to induce humoral responses after i.p. immunization. In fact, using an improved invertible promoter system that results in much higher CtxB levels, specific antibodies were also induced by i.p. immunization (manuscript in preparation).

At present we are evaluating the immune responses to a number of HIV-1 and FIV-antigens expressed in SL3261 by this improved invertible promoter system.

\section{ACKNOWLEDGEMENTS}

The authors are grateful to Geert van Amerongen, Herman Näring, Nico Schmidt and $A b$ van Arnhem of the Animal Facilities Department for animal handling, and to Conny Kruyssen for preparing the manuscript. This investigation was supported by a grant of the 'Raad voor Gezondheidsonderzoek', The Hague, The Netherlands (grant number 88-108/89027).

\section{REFERENCES}

1 Dougan, G. and Maskell, C.E. Live attenuated Salmone/la vaccines as carriers of antigens to the secretory and systemic immune system. In: Vaccination Strategies of Tropical Diseases (Ed Liew, F.Y.) CRC Press, Boca Raton, FA, 1991, pp. 48-64

2 Aggarwal, A., Kumar, S., Jaffe, R., Hone, D., Gross, M. and Sadoff, J. Oral Salmonella: Malaria circumsporozoite recombinants induce specific CD8 + cytotoxic T cells. J. Exp. Med. 1990, 172, 1083-1090

3 Flynn, J.L., Weiss, W.R., Norris, K.A., Siefert, H.S., Kumar, S. and So, M. Generation of a cytotoxic T-lymphocyte response using a Salmonella antigen-delivery system. Mol. Microbiol. 1990, 4, 2111-2118

4 Tite, J.P.. Gao, X.M., Hughes-Jenkins, C.M., Lipscombe, M., $O^{\prime}$ Callaghan, D. and Dougan, G. Antiviral immunity induced by recombinant nucleoprotein of influenza-A virus. III. Delivery of recombinant nucleoprotein to the immune system using attenuated Salmonella typhimurium as a live carrier. Immunology 1990, 70 , $540-546$

5 Poirier, T.P., Kehoe, M.A. and Beachey, E.H. Protective immunity evoked by oral administration of attenuated aroA Salmonella typhimurium expressing cloned streptococcal $M$ protein. J. Exp. Med. 1988, 168, 25-32

6 Sadoff, J.C., Ballou, W.R., Baron, L.S., Majarian, W.R., Brey, R.N., Hockmeyer, W.T. et al. Oral Salmonella typhimurium vaccine expressing circumsporozoite protein protects against malaria. Science 1988, 240, 336-338

7 Wadhan, M.H., Sérié, C., Cerisier, Y., Sallam, S. and Germanier, R A controlled field trial of live Salmonella typhi strain Ty21a ora vaccine against typhoid: three-year results. J. Infect. Dis. 1982, 145 292-295 
8 Levine, M.M., Ferreccio, C., Cryz, S. and Ortiz, E. Comparison of enteric-coated capsules and liquid formulation of Ty21A typhoid vaccine in randomised controlled field trial. Lancet 1990, 336 , 891-894

9 Tacket, C.O., Hone, D.M., Curtiss, R. III, Kelly, S.M., Losonsky, G., Guers, L. et al. Comparison of the safety and immunogenicity of delta aroC delta aroD and delta cya delta crp Salmonella typhi strains in adult volunteers. Infect. Immun. 1992, 60, 536-541

10 Yan, Z.X., Reuss, F. and Meyer, T.F. Construction of an invertible DNA segment for improved antigen expression by a hybrid Salmonella vaccine strain. Res. Microbiol. 1990, 141, 1003-1004

11 Hoiseth, S.K. and Stocker, B.A.D. Aromatic-dependent Salmonella typhimurium are non-virulent and effective as live vaccines. Nature 1981, 291, 238-239

12 Sambrook, J., Fritsch, E.F. and Maniatis, T. Molecular cloning. A Laboratory Manual, Cold Spring Harbor Laboratory Press, New York, 2nd edn, 1989

13 Laemmli, U.K. Cleavage of structural proteins during the assembly of the head of bacteriophage T4. Nature 1970, 227, 680-685

14 Plasterk, R.H.A., Kanaar, R. and Van de Putte, P. A genetic switch in vitro: DNA inversion by Gin protein of phage Mu. Proc. Natl Acad. Sci. US A 1984, 81, 2689-2692

15 Kahmann, R., Rudt, F. and Mertens, G. Substrate and enzyme requirements for in vitro site specific recombination in bacteriophage Mu. Cold Spring Harbor Symp. Quant. Biol. 1984, 49, 285-294

16 Remaut, E., Stanssens, P. and Fiers, W. Plasmid vectors for high-efficiency expression controlled by the $P_{L}$ promoter of coliphage lambda. Gene 1981, 15, 81-93

17 Holmgren, J. Actions of cholera toxin and the prevention and treatment of cholera. Nature 1981, 292, 413-417

18 Elson, C.O. and Ealding, W. Generalized systemic and mucosa immunity in mice after mucosal stimulation with cholera toxin. J. Immunol. 1984, 132, 2736-2741

19 Molina, C.N. and Parker, C.D. Murine antibody response to ora infection with live aroA recombinant Salmonella dublin vaccine strains expressing filamentous hemagglutinin antigen from Bordetella pertussis. Infect. Immun. 1990, 58, 2523-2528

20 Maskell, D.J., Sweeney, K., O'Callaghan, D., Hormaeche, C.E., Liew, F.H. and Dougan, G. Salmonella typhimurium aroA mutants as carriers of the Escherichia coli heat-labile enterotoxin B subunit to the murine secretory and systemic immune systems. Microb. Pathog. 1987, 2, 211-221

21 Connell, N., Stover, C.K. and Jacobs, W.R. Old microbes with new faces: molecular biology and the design of new vaccines. Curr. Opin. Immunol. 1992, 4, 442-448

22 Curtiss, R. III, Kelly, S.M., Gulig, P.A. and Nakayama, K. Selective delivery of antigens by recombinant bacteria. Curr. Top. Microbiol. Immunol. 1989, 146, 35-49

23 Curtiss, R. III, Nakayama, K. and Kelly, S.M. Recombinant avirulent Salmonella vaccine strain with stable maintenance and high level expression of cloned genes in vivo. Immunol. Invest. 1989, 18, 583-596

24 Nakayama, K., Kelly, S.M. and Curtiss, R. Construction of an asd ${ }^{+}$ expression-cloning vector: Stable maintenance and high level expression of cloned genes in a Salmonella vaccine strain. Biotechnology 1988, 6, 693

25 Hone, D., Attridge, S., Van den Bosch, L. and Hackett, J. A chromosomal integration system for stabilization of heterologous genes in Salmonella based vaccine strains. Microb. Pathog. 1988, 5, 407-418

26 Strugnell, R.A., Maskell, D., Fairweather, N., Pickard, D., Cockayne, A., Penn, C. and Dougan, G. Stable expression of foreign antigens from the chromosome of Salmonella typhimurium vaccine strains. Gene 1990, 88, 57-63

27 Stueber, D. and Bujard, H. Transcription from efficient promoters can interfere with plasmid replication and diminish expression of plasmid specified genes. EMBO J. 1982, 1, 1399-1404

28 Mitov, I., Denchev, V. and Linde, K. Humoral and cell-mediated immunity in mice after immunization with live oral vaccines of Salmonella typhimurium: auxotrophic mutants with two attenuating markers. Vaccine 1992, 10, 61-66 